\title{
Focus on emergency general surgery
}

\author{
Fernando Turégano ${ }^{1,2}$
}

(c) The Author(s), under exclusive licence to Springer-Verlag GmbH Germany 2022

In this Focus-on issue on emergency general surgery-EGS, several studies have addressed common problems and some controversies that are still too relevant to the practice of EGS. They cover aspects such as the increasing importance of an adequate management program for the specialty, the search for a better prediction score for those patients with the all too frequent condition of small bowel obstruction-SBO in need of timely surgical management, an ESTES 'snapshot audit' of practice in complicated acute biliary calculous disease, the results of omental patch repair of large-size perforations of gastroduodenal ulcers, the feasibility of routine avoidance of postoperative NG tube decompression after acute SBO surgery, and, finally, the most appropriate open abdomen closure methods for severe abdominal sepsis.

Eaton et al. [1] from the Adams Cowley Shock Trauma Center, remind us that the evolution of EGS carries with it the need to acknowledge that quality outcomes, performance improvement and research are paramount in delivering the best care. They describe the maturation of their EGS quality program with the novel role of Service-Based Advanced Practice Providers (SB APP) who provide administrative oversight in the capacity of an EGS Program Manager. In 2009, they formalized the Division of Acute Care Emergency Surgery (ACES) as a separate and distinct clinical service. Any acute general surgery admission was accepted by the ACES service via the emergency department (ED), internal consultation or intra-hospital transfers, with some exceptions. In 2017, a separate ACES quality structure was formalized with primary focus on scheduled quality meetings, morbidity and mortality peer review, outcomes review and performance improvement (PI) initiatives. Theirs is probably the first medical center to describe an SB APP practicing in the role of a Program Manager within a dedicated

Fernando Turégano

fernando.turegano@salud.madrid.org

1 University General Hospital Gregorio Marañón-UGHGM, Madrid, Spain

2 University Complutense of Madrid, Madrid, Spain
EGS service. Their most substantial finding was that the ACES census was drastically underrepresented, giving the impression of a small service with a low yearly admission rate. This led to a prompt review of the way new admissions were coded within the hospital EMR system, and an enhanced logic was created. They state that the EGS population accounts for more than 3 million admissions a year in the US, has a higher mortality and complication rates than general surgery patients, and the potential impact of improving outcomes is substantial in both the economic and social sense. Their conclusion is that the quest to improve quality of care for the EGS patient requires timely review of highquality, accurate data by dedicated and trained personnel.

Berge et al. [2] from the Centre Hospitalier Universitaire d'Angers, in France, have developed a Multi Detector Computed-Tomography (MDCT) score to predict single band adhesion (SBA) versus matted adhesions (MA) as as an etiology of SBO, thus leading to earlier surgical intervention in SBA, and with a more frequent and safer use of laparoscopy. Interestingly, they found that if the score was $\geq 7$, the probability of the mechanism of SBO being SBA was of $100 \%$. Theirs is a first step toward the improvement of SBO management through selecting SBA patients who would benefit from immediate, semi-emergent or elective surgery because of their risk of medical treatment failure, their probability of success from laparoscopy, and the lower risk of recurrence as compared to MA. Indeed, a very interesting study with beneficial potential implications for that frequent condition.

Bass et al. and the ESTES Cohort Studies Collaborative Group [3] describe the results of a 'snapshot audit' performed across Europe in late autumn/winter 2018, to better define the epidemiology, management, and outcomes in patients with Acute Complicated Calculous Biliary Disease (ACCBD). Data were recorded contemporaneously and stored on a secure, user-encrypted online platform (REDCap®) without patient-identifiable information. The primary outcome measure was index admission surgical definitive treatment. The secondary outcome measures were length of stay, the postoperative major complication rate defined as Clavien-Dindo classification, the postoperative length of 
stay, and the postoperative mortality rate. A dedicated Acute Care/Emergency Surgery (ACS) service line (separate from elective General Surgery) existed only in 7 (28\%) centers included, indicating the need to further proceed with the implementation of these dedicated services throughout the European Union and elsewhere. Although highly recommended by several different guidelines and societies, only $50 \%$ of patients followed by this study underwent cholecystectomy during the index hospitalization. Another observation from this study was that centers employing an ACS service line (compared to the traditional 'on call' model of care provided by an elective General Surgery service) had a decreased time to intervention, that could lead to decreased length of post-operative hospital stay. The results of this study should make an interesting reading for all, and prompt further international collaborative efforts in many aspects of our specialty.

Chang et al. [4] from the Tan Tock Seng Hospital in Singapore have aimed to compare the outcomes of patients with perforated peptic ulcers-PPU of $\geq 20 \mathrm{~mm}$ undergoing gastrectomy, with those sujected to a simple omental patch repair. They rightfully state that, in surgery, sometimes "less is better", especially in frail and elderly patients, and one has to resist the temptation of being more aggressive for the sake of the patient, and disregard possible criticism at the M\&M rounds if things do not turn out right for the patient at the end. It is true that one is tempted to do more in large ulcers, since a simple Graham patch repair does not seem enough. It is noteworthy that almost half of their patients had giant ulcers (PPU $\geq 30 \mathrm{~mm}$ ), and subgroup analysis of these patients did not show statistically significant differences in intra-abdominal collection, post-operative leakage, re-operation and all-cause mortality between the two groups. Their conclusion was that omental patch repair confers similar perioperative outcomes as compared to gastrectomy in patients with large PPU. Only one patient underwent laparoscopy, and one wonders why a laparoscopic approach was not more widely used. Comparable demographics and clinical profile across both arms in the study, and the use of multivariate analysis to account for confounding variables strengthen the comparison between the two techniques which has been lacking in current literature. This should be a landmark reference paper which adds useful information in the management of this, sometimes, technical challenge.

Delestre et al. [5], again from the Centre Hospitalier Universitaire d'Angers, in France, have focused on the safety and feasibility of early removal of nasogastric tube (NGT) after SBO surgery, and its likely influence on enhanced recovery after surgery (ERAS) protocols after this surgery. Some have reported that the need for surgery or the morbidity of the treatment were not significantly reduced using NGT in non-surgical treatment of acute SBO. Also, after emergency or elective surgery, NGT decompression has not proven its interest either, but no study has specifically assessed it in acute SBO surgery. The first outcome measure was the occurrence of severe morbidity, and the secondary outcome measures were the occurrence of overall morbidity, postoperative ileus (POI), and the occurrence of a postoperative pneumonia. NGT replacement was required in $16.5 \%$ of delayed removal patients vs. $11.5 \%$ in early removal patients. Mean time of length of stay was significantly longer in the delayed removal group than in the early removal group. In multivariate analysis, POI was significantly associated with postoperative NGT but it was independent from surgical access, diameter of the small bowel and the risk factors for a complicated procedure. In addition to be associated with a reduction of POI occurrence and the absence of a severe postoperative complication increase, early NGT removal was not associated with an increase of NGT replacement. The time to surgery was the only parameter significantly associated with postoperative morbidity, advocating for the fact that NGT is not the only factor improving postoperative course, as we all know. They conclude that early NGT removal after acute SBO surgery seemed to be feasible, safe and efficient, at least in selected patients. This primary study is the first foundation stone in building the implementation of ERAS protocols after this surgery.

Poillucci et al. [6], from the Policlinico di Roma, present the results of a retrospective study addressing the effects of different abdominal closure methods and their outcomes in patients presenting with abdominal sepsis treated with open abdomen-OA. They compare intra-hospital morbidity, mortality, and Definitive Fascial Closure (DFC) rates in those patients who underwent OA with temporary Negative Pressure Wound Therapy (NPWT) versus the Bogota-Bag closure technique. They also quantified the impact of NPWT use on surgeons' decisions to perform a primary colonic anastomosis or an end colostomy for patients with sepsis of hindgut-origin. Disease severity was measured using the Mannheim Peritonitis Index (MPI) score and the APACHEII score on admission. Several strategies for maintaining the OA have indeed been reported during the last two to three decades, and they result in different DFC rates and enteroatmospheric fistula risks. In their study, NPWT results as the best temporary abdominal closure technique to decrease mortality and colostomy rates in patients managed with OA for severe intra-abdominal infections. The study has the merit of being focused on the use of OA in a large cohort of patients with severe IAIs, thus providing useful information in a field of research monopolized by trauma reports.

\section{Declarations}

Conflict of interest I have no conflicts of interest with any of the groups or topics discussed. 


\section{References}

1. Eaton B, O’Meara LB, Aresco C, Scalea ThM, Diaz JJ, Bruns BR. The Evolution of Emergency General Surgery - It's Time for a Dedicated Program Manager. 2020;3:1-7. https://link.springer. com/article/10.1007/s00068-020-01475-1

2. Berge P, Delestre M, Paisant A, Hamy A, Aubé Ch, Hamel J-F, Venara A. Diagnosis of single adhesive bands versus matted adhesions in small bowel obstructions: a radiological predictive score. 2021;8:1-0. https://link.springer.com/article/10.1007/ s00068-020-01580-1

3. Bass GA, Gillis AE, Cao Y, Mohseni Sh, ESTES Cohort Studies Collaborative Group. Patterns of prevalence and contemporary clinical management strategies in complicated acute biliary calculous disease - an ESTES 'snapshot audit' of practice. 2020;7:1-3. https://link.springer.com/article/10.1007/s00068-020-01433-X
4. Chan KS, Wang YL Chan XW, Shelat VG. Outcomes of omental patch repair in large or giant perforated peptic ulcer are comparable to gastrectomy. 2021;47(6):1745-1752. https://link.springer. com/article/10.1007/s00068-019-01237-8

5. Delestre M, Berge P, Aubé Ch, Hamy A, Hamel JF, Paisant A, Venara A. Nasogastric Tube After Small Bowel Obstruction Surgery could be avoided: A Retrospective Cohort Study. 2020;23:17. https://link.springer.com/article/10.1007/s00068-020-01529-4

6. Poillucci G, Podda M, Russo G, Perri SG, Ipri D, Manetti G, Lolli MG, De Angelis R. Open abdomen closure methods for severe abdominal sepsis: a retrospective cohort study. 2021;47(6):1819-1825. https://link.springer.com/article/10.1007/ s00068-020-01379-0 